\title{
Evaluation of ten commercial blood culture systems to isolate a pyridoxal-dependent streptococcus
}

\section{GS TILLOTSON*}

\section{From the Department of Bacteriology, Tameside General Hospital, Ashton-under-Lyne, Lancs}

SUMMARY This study evaluated the ability of ten commercial blood cultures to support the growth of a nutritional variant Streptococcus mitior (pyridoxal-dependent). The abilities of two established and two new agar formulations are also reported. The dependable isolation of a fastidious streptococcus can best be obtained with fastidious anaerobe broth (FAB) (Lab M Ltd, Ford Lane, Salford) for blood cultures in conjunction with one of the new media. FAB agar with the addition of heated blood was found to support the growth of pyridoxal-dependent Strep mitior and other fastidious organisms.

A few cases of endocarditis evade the most earnest attempts to isolate causative organisms. There are several possible explanations for this problem: (a) that bacteria are sequestered within vegetations on the heart valves, (b) the patient has had previous antibiotic treatment, (c) the endocarditis is of nonbacterial origin-for example, Coxiella burneti or Coxsackie B virus and (d) the endocarditis is due to fastidious organisms which are difficult to isolate. In this last category come Brucella spp, Haemophilus spp; ${ }^{1}$ Streptobacillus moniliformis; L-forms ${ }^{2} 3$ and vitamin $\mathbf{B}_{6}$ (pyridoxal)-dependent streptococci. ${ }^{4}$ The nutritionally variant strains of streptococci have been implicated in cases of endocarditis. ${ }^{4-6}$ Carey $e t$ $a l .{ }^{5}$ showed that the addition of either pyridoxal hydrochloride or cysteine induced growth in three strains of Strep mitior. ${ }^{7}$ Pyridoxal is a cofactor required in the biosynthesis of cysteine ${ }^{8}{ }^{9}$ and thiol compounds. ${ }^{10}$

Roberts et al. ${ }^{4}$ studied the incidence of pyridoxaldependent streptococci in cases of endocarditis in two periods at the New York Hospital. In the periods $1944-55$ and 1970-8, they isolated 12 strains of pyridoxal-dependent Strep mitior, which accounted for $5-6 \%$ of microbial endocarditis cases. McCarthy" and Carey ${ }^{6}$ state "that blood cultures have to be supplemented by pyridoxal hydrochloride, pyridoxamine dihydrochloride or L-cysteine ${ }^{13}$ in order for these variants to grow." This study evaluates four broth culture media and ten commercially available non-supplemented blood cultures in their abilities to culture this fastidious organism. Also a

*Present address: Department of Microbiology, Hope Hospital, Salford.

Accepted for publication 3 December 1980 series of solid media are assessed for their abilities to grow the pyridoxal-dependent Strep mitior.

\section{Material and methods}

The "pyridoxal-dependent" Strep mitior (ref R/80/ 2345) was obtained from Dr G Colman, Public Health Laboratory, Colindale, London.

Assessment of basic liquid support media

Four different media were tested:

(1) Brain heart infusion (BHI) broth (Lab M, Salford, England-Lab 49).

(2) Fastidious anaerobe broth (FAB) (Lab M, Salford, England-Lab 71).

(3) Todd-Hewitt broth (Difco, Detroit, Michigan -0492-97).

(4) Nutrient broth (Oxoid, Basingstoke, England -CM-1).

All four media were assessed for their ability to grow the reference strain under three different conditions (a) with added pyridoxal hydrochloride (Koch-Light Ltd) $100 \mu \mathrm{g} / \mathrm{ml}$; (b) with $10 \%$ fresh human blood and (c) without any supplementation.

Each of the 12 tubes was inoculated with approxi- O mately 100 colony forming units (CFU) of the $N$ pyridoxal-dependent streptococcus and incubated at $37^{\circ} \mathrm{C}$ in $10 \% \mathrm{CO}_{2}$ (in a $\mathrm{CO}_{2}$ controlled incubator) for $24 \mathrm{~h}$ and then examined for growth by inspection and also by subculturing on to the following different media:

(a) FAB heated blood agar-Lab 71.

(b) FAB blood agar-Lab 71.

(c) Columbia heated blood agar (Oxoid)-CM 331.

(d) Columbia blood agar (Oxoid)—CM 331. 
Included in these media was an agar with FAB (Lab M) as a base, with either whole or heated horse blood (Gibco) as an additive. The rationale for comparing whole and heated blood was to show any differing constituents released on the heating of blood.

A standard sterile plastic loop of $10 \mu \mathrm{l}$ volume (Nunc, Denmark) was used to inoculate the $24 \mathrm{~h}$ broth cultures on to each medium. Filter paper discs ( $5 \mathrm{~mm}$ diam) containing approximately $40 \mu \mathrm{g} / \mathrm{ml}$ pyridoxal-hydrochloride were also placed on these plates.

\section{Assessment of commercial blood culture sets}

Roberts et al. ${ }^{4}$ suggest that supplementation of blood culture sets is necessary to support the growth of pyridoxal-dependent streptococci. Thus 10 commercially available blood culture systems were evaluated in their ability to support these organisms. The media used were:

(i) Oxoid (Basingstoke, England)-Hartley's digest broth-PM 1185.

(ii) Oxoid (Basingstoke, England)-Schaedler broth-CM 497.

(iii) Difco (Detroit, Michigan)-BHI-0652-37.

(iv) Difco (Detroit, Michigan)_-Thiol broth0355-74.

(v) Medical Wire Equipment (Corsham, Wilts) -Vacuneda-MW BC 8.

(vi) Gibco (Paisley, Scotland)-Thioglycollate + resazurin-B-6110.

(vii) Gibco (Paisley, Scotland)-BHI brothB-6031.

(viii) Gibco (Paisley, Scotland)—Columbia broth -B-6050.

(ix) Lab M (Salford, England)-FAB-Lab 71.

(x) Lab M (Salford, England)-BHI brothLab 49.

The reference organism was inoculated at 2 different concentrations $10^{6} \mathrm{CFU} / \mathrm{ml}$ and $10^{4} \mathrm{CFU} / \mathrm{ml}$ into the blood culture bottles; each set was supplemented with fresh human blood (approximately $1 / 10$ dilution) as in normal clinical methods. Each set was incubated at $37^{\circ} \mathrm{C}$ in $10 \% \mathrm{CO}_{2}$ in an incubator for $24 \mathrm{~h}$ and then subcultured with a standard sterile plastic loop of $10 \mu$ l volume on to FAB-based agar containing either whole or heated blood. The FAB whole blood agar plates were incubated at $37^{\circ} \mathrm{C}$ in anaerobic conditions (Raven scientific cabinet) for $24 \mathrm{~h}$ and the FAB-heated blood agar plates were incubated at $37^{\circ} \mathrm{C}$ in $10 \% \mathrm{CO}_{2}$ in a controlled incubator. The subculturing was performed under a Microflow lamina flow hood. The plates were examined for growth and recorded. The blood cultures were reincubated for a further $48 \mathrm{~h}$ and then sub- cultured on to FAB agar which were incubated as before.

DETERMINATION OF THE ABILITY OF FAB AGAR TO SUPPORT FASTIDIOUS ORGANISMS To determine whether FAB heated blood agar would support the growth of other fastidious organisms, the following strains were tested on both whole blood and heated blood versions of FAB agar and also Columbia heated blood agar using $10 \mu \mathrm{l}$ aliquots of $24 \mathrm{~h}$ broth cultures.

(a) Streptococcus mitior pyridoxal-dependent

(b) Streptococcus milleri

(c) $\beta$-haemolytic streptococcus Lancefield Group A

(d) Neisseria gonorrhoeae

(e) Neisseria meningitidis

(f) Haemophilus influenzae

(g) Bacteroides melaninogenicus

(h) Peptostreptococcus anaerobius

(i) Escherichia coli

(j) Pseudomonas aeruginosa

(k) Staphylococcus aureus

All strains except $(a)$ were clinical isolates. The whole blood FAB plates were incubated anaerobically in a Raven scientific anaerobic cabinet at $37^{\circ} \mathrm{C}$ whilst the FAB and Columbia based heated blood agar plates were incubated at $37^{\circ} \mathrm{C}$ in $10 \% \mathrm{CO}_{2}$.

\section{Results and discussion}

Roberts et al. ${ }^{4}$ have shown that pyridoxal-dependent Strep mitior is difficult to isolate without media supplementation but pyridoxal addition would seem unnecessary as human blood usually contains $20-45$ $\mu \mathrm{g} / \mathrm{ml}$ of this essential cofactor. ${ }^{12}$ The problem lies in the ability to support the growth of these nutritional variants on solid media.

\section{BASIC LIQUID SUPPORT MEDIA}

These were tested both with and without pyridoxal hydrochloride, and with fresh human blood. Their abilities to support the growth of the test organism are shown in Table 1. The unsupplemented BHI, Todd-Hewitt, and Nutrient broths were all unable to support the fastidious Strep mitior, whereas the unsupplemented FAB supported profuse growth. The addition of $100 \mu \mathrm{g} / \mathrm{ml}$ pyridoxal hydrochloride enables the BHI broth to support the organism, while supplementation of FAB did not alter its growthsupporting performance. Pyridoxal-enriched ToddHewitt broth and Nutrient broth did not support the fastidious organism. This is contrary to Roberts $e t$ al. ${ }^{4}$ who used supplemented Todd-Hewitt broth to isolate and passage the organism; the reason for this difference is unclear. The addition of fresh human 
Table 1 Assessment of basic liquid support media (tube assay)

\begin{tabular}{llll}
\hline Medium (broth) & Control & $\begin{array}{c}+ \text { Pyridcxal } \\
\text { hydrochloride }\end{array}$ & $\begin{array}{l}+ \text { Fresh human } \\
\text { blood }\end{array}$ \\
\hline Brain heart infusion & - & - & + \\
Fastidious anaerobe & +++ & +++ & +++ \\
Todd-Hewitt & - & - & + \\
Nutrient & - & - & + \\
\hline
\end{tabular}

- = no growth.

$=$ slight growth (10-20 colonies).

$=$ moderate growth (20-100 colonies).

$t+t=$ heavy growth ( $>100$ colonies)

blood (approximately $10 \%$ ) to each of the four test broths enables them all to support the pyridoxaldependent Strep mitior, so the addition of blood normally provides adequate supplementation. FAB performs much better than the other three test media under the three different conditions.

The four different media were subcultured on to four different solid media to assess whether routine methods were adequate, or, whether a new medium was required. As can be seen from Table 2, the normal Columbia (Oxoid) blood agar would support the test organism only when supplemented by the addition of a filter paper disc, impregnated with approximately $40 \mu \mathrm{g} / \mathrm{ml}$ of pyridoxal hydrochloride, on this medium the streptococcus grew in a zone of 38 $\mathrm{mm}$ diameter. Using Columbia (Oxoid) heated blood agar the same occurred except that the zone was 45 $\mathrm{mm}$ diameter. Strep mitior grew on both FAB blood agar basal medium and in the presence of a pyridoxal disc, in which case growth was enhanced around the disc. FAB heated blood agar allowed uniform growth of the organism across the plate and no growth enhancement was noticeable around the disc. Thus the new formulation agar alone was sufficient to support the growth of this fastidious organism from unsupplemented FAB.

COMMERCIAL BLOOD CULTURE ASSESSMENT

Ten commercially available blood culture media were evaluated; all were tested at two different

Table 2 Solid media subculture

\begin{tabular}{lll}
\hline Agar & Basal medium & + Pyridoxal disc \\
\hline Columbia blood & - & $+(38 \mathrm{~mm}$ zone $)$ \\
Columbia heated blood & - & $+(45 \mathrm{~mm}$ zone $)$ \\
FAB blood & ++ & $+++*$ \\
FAB heated blood & +++ & $+++\dagger$ \\
\hline
\end{tabular}

- = no growth.

$+=$ slight growth (10-20 colonies)

$++=$ moderate growth (20-100 colonies).

$+++=$ heavy growth ( $>100$ colonies).

FAB $=$ fastidious anaerobe broth.

*Zone of enhanced growth.

+ No difference in growth around disc.

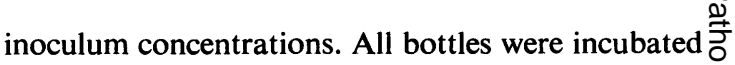
under identical conditions and subcultured on to the same media. The medium used was a solid form of $\overrightarrow{\overrightarrow{\vec{p}}}$ FAB (Lab M) containing either whole or heated blood. The results of subculturing the blood culture등 sets at $24 \mathrm{~h}$ and $72 \mathrm{~h}$ incubation can be seen in Table $\overline{\bar{\omega}}$ 3 . The major conclusion from these results is that $\overline{\mathrm{D}}$ media normally used to isolate anaerobic bacteria are adequate for the growth of this organism. All the $e^{\infty}$ anaerobic broths, Schaedler broth (Oxoid), thiol $\vec{O}$ broth (Difco), thioglycollate broth (Gibco) and FAB $\vec{A}$ $(\mathrm{Lab} \mathrm{M})$ are excellent support systems for pyridoxal- $\omega$ dependent streptococci. The aerobic media produced variable results. BHI broth (all makes) showed ${ }_{\omega}^{\circ}$ growth but not as quickly or as profusely as the + anaerobic media. Columbia broth, Hartley's digest ${ }_{i}^{\infty}$ broth and Vacuneda were all comparatively poore్ growth media.

All the media, except FAB and thiol broth, showed no viable bacteria after $72 \mathrm{~h}$ incubation; this is probably due to the glucose degradation and the resultant acidity. The reason for the prolonged $\frac{c}{\infty}$ growth in FAB is due to the low level of fermentable carbohydrate; the absence of glucose in particular. $\mathscr{\infty}$ The reason for the prolonged viability in thiol broth. is not known.

The inoculum concentration seems to have little effect on the ability of the organism to grow and also the time taken before growth can be seen. Using a气 range of inoculum concentrations from $10-10^{8} \stackrel{\square}{\Omega}$ CFU/ml, FAB blood culture systems without added $\underset{F}{\Rightarrow}$ human blood were inoculated and incubated at $37^{\circ} \mathrm{C}$. The bottles were examined after $24 \mathrm{~h}$. Growth was visible to some extent in all bottles and this waso substantiated on subculturing on to FAB heated blood agar. Thus the ability of FAB blood cultures? to support the growth of 1-10 colonies per $\mathrm{ml}$ of pyridoxal-dependent Strep mitior was shown. Providing the correct blood cultures were taken, it is unlikely that a case of endocarditis due to the organism would escape notice.

\section{ABILITY OF FAB AGAR TO SUPPORT OTHER ORGANISMS}

Before using FAB based agar routinely, other equally? fastidious and non-fastidious organisms were tested 0 under normal conditions to assess its growth- $\omega$ supporting qualities. The range of organisms (see Methods) were tested on both whole blood or heatedo blood FAB media; for results see Table 4. All the common organisms, fastidious and non-fastidious, were able to grow in an easily recognisable form on $\frac{7}{0}$ at least one type of the medium.

The FAB heated blood agar is recommended for $\stackrel{\mathbb{D}}{\mathscr{\mathbb { P }}}$ its ability to support pyridoxal-dependent strepto- $\stackrel{\mathbb{Q}}{\varrho}$ cocci. The subcultures of the FAB blood cultures 
Table 3 Pyridoxal-dependent streptococcus in commercial blood culture sets (subcultured on to FAB-based agar with either whole or heated blood)

\begin{tabular}{|c|c|c|c|c|c|c|c|c|}
\hline \multirow[t]{3}{*}{ Medium (broth) } & \multicolumn{4}{|l|}{$10^{\circ}$ inoculum } & \multicolumn{4}{|l|}{$10^{4}$ inoculum } \\
\hline & \multicolumn{2}{|l|}{$24 h$} & \multicolumn{2}{|l|}{$72 h$} & \multicolumn{2}{|l|}{$24 h$} & \multicolumn{2}{|l|}{$72 h$} \\
\hline & *Heated blood & †Blood & *Heated blood & $†$ Blood & *Heated blood & †Blood & *Heated blood & †Blood \\
\hline (i) Oxoid (Hartley) & + & - & $+t$ & - & + & + & - & - \\
\hline (ii) Oxoid (Schaedler) & ++ & +++ & - & - & ++ & $+t+$ & - & - \\
\hline (iii) Difco (BHI) & $+t$ & +++ & - & - & $+t+$ & ++ & - & - \\
\hline (iv) Difco (thiol) & +++ & +++ & + & - & $+t+$ & $++t$ & + & - \\
\hline (v) MWE (Vacuneda) & + & ++ & - & - & + & ++ & - & - \\
\hline (vi) Gibco (thioglycollate) & ++ & $+t+$ & - & - & ++ & +++ & - & - \\
\hline (vii) Gibco (BHI) & + & + & - & - & + & + & - & - \\
\hline (viii) Gibco (Columbia) & + & +++ & - & - & + & + & - & - \\
\hline $\begin{array}{l}\text { (ix) Lab M (FAB) } \\
\text { (x) Lab M (BHI) }\end{array}$ & $\begin{array}{l}+t+ \\
+\end{array}$ & $\begin{array}{l}+++ \\
+++\end{array}$ & $\begin{array}{l}+++ \\
-\end{array}$ & $\begin{array}{l}++ \\
-\end{array}$ & $\begin{array}{l}+++ \\
+\end{array}$ & $\begin{array}{l}++t \\
++t\end{array}$ & $\begin{array}{l}+t+ \\
-\end{array}$ & $\frac{t+}{-}$ \\
\hline
\end{tabular}

- = no growth.

$+=$ slight growth (10-20 colonies).

$++=$ moderate growth (20-100 colonies).

$+++=$ heavy growth ( $>100$ colonies).

BHI = brain heart infusion.

FAB = fastidious anaerobe broth.

* incubated in $10 \% \mathrm{CO}_{2}$ at $37^{\circ} \mathrm{C}$.

tincubated anaerobically at $37^{\circ} \mathrm{C}$.

Table 4 Assessment of FAB agar as a growth medium to support various organisms

\begin{tabular}{llll}
\hline & FAB & & $\begin{array}{l}\text { Columbia } \\
\text { *Heated } \\
\text { blood }\end{array}$ \\
\cline { 2 - 3 } & $\begin{array}{l}\text { *Heated } \\
\text { blood }\end{array}$ & + Blood & \\
\hline Strep mitior B $_{6}$ dep & +++ & + & - \\
Strep milleri & +++ & +++ & ++ \\
Group A streptococcus & +++ & +++ & +++ \\
Neisseria gonorrhoeae & ++ & + & +++ \\
N meningitidis & ++ & + & +++ \\
Haem influenzae & ++ & + & +++ \\
Bact melaninogenicus & - & +++ & - \\
Peptostrep anaerobius & - & ++ & - \\
E coli & +++ & +++ & +++ \\
Ps aeruginosa & ++ & + & ++ \\
Staph aureus & +++ & +++ & +++ \\
\hline
\end{tabular}

- = no growth.

$+=$ slight growth (10-20 colonies).

$++=$ moderate growth (20-100 colonies).

$++t=$ heavy growth $(>100$ colonies $)$

*incubated in $10 \% \mathrm{CO}_{2}$ at $37^{\circ} \mathrm{C}$.

tincubated anaerobically at $37^{\circ} \mathrm{C}$.

containing Strep mitior on to FAB heated blood agar had a characteristic blue irridescent sheen which was rarely seen with other viridans streptococci. The reason for this appearance is not known.

The importance of isolating fastidious organisms which may be implicated in endocarditis is obvious. The use of specially selective or supplemented media is necessary but only up to the point where the process is efficient and manageable. This study demonstrated that most commercial broth blood culture systems are capable of supporting the growth of pyridoxal-dependent streptococci. It is important also that these organisms can be easily grown on solid media so it is up to the individual laboratory to ascertain whether they can isolate and culture this important and nutritionally variant organism. After the work carried out so far, the use of Lab M's FAB blood cultures in conjunction with FAB heated blood agar allows easy and dependable isolation of these fastidious organisms without any supplementation; it can be seen that this medium may replace Columbia heated blood agar without any detrimental effects.

I would like to thank Mr MW Buckley for excellent technical assistance, Mrs W O'Hare and Dr PF Unsworth for useful discussions and Mrs B Hyde for typing this script.

\section{Appendix}

Composition of fastidious anaerobe broth (Lab MLab 71)

\begin{tabular}{ll}
\hline Constituent & $g / l$ \\
\hline Lab M special peptone & $15 \cdot 0$ \\
Lab M yeast extract & $10 \cdot 0$ \\
Sodium thioglycollate & $0 \cdot 5$ \\
Sodium chloride & $2 \cdot 5$ \\
Cysteine hydrochloride & 0.5 \\
Haemin & 0.005 \\
Vitamin K & 0.0005 \\
Resazurin & $0 \cdot 001$ \\
Sodium bicarbonate & $0 \cdot 4$ \\
Lab M agar No 1 & $0 \cdot 75$ \\
pH 7.2 & \\
\hline
\end{tabular}

Fastidious anaerobe agar

Constituents: FAB (Lab 71) 29 g/l 


\section{Lab M agar No 2 (LC 2) $\quad 10 \mathrm{~g} / \mathrm{l}$ Distilled water \\ 1.01}

Boil, autoclave for $15 \mathrm{~min}$ at $15 \mathrm{psi}$, then add $50 \mathrm{ml}$ of defibrinated sterile horse blood (Gibco) and heat. When the medium has cooled to $45^{\circ} \mathrm{C}$, dispense aseptically.

\section{References}

${ }^{1}$ Hodge JLR, Bremner DA. Haemophilus endocarditis and the isolation of haemophilic bacteria in blood culture: case report. NZ Med J 1974;512:824-5.

${ }^{2} \mathrm{Neu} \mathrm{HC}$, Goldreyer B. Isolation of protoplasts in a case of enterococcal endocarditis. Am J Med 1968;45:784-8.

${ }^{3}$ Mattman AH, Mattman PE. L-forms of Strep faecalis in septicaemia. Arch Intern Med 1965;115:315-21.

- Roberts RB, Krieger AG, Schiller NL, Gross KC. Viridans streptococcal endocarditis; the role of various species including pyridoxal-dependent streptococci. Rev Infect Dis 1979;1:955-65.

${ }^{5}$ Carey RB, Brause BD, Roberts RB. Antimicrobial therapy of vitamin $B_{6}$-dependent streptecoccal endocarditis. Ann Intern Med 1977;87:150-4.

- Carey RB. Culture negative endocarditis. Lancet 1978; ;
278.

7 Colman G, Williams REO. Taxonomy of some human virdans streptococci. In: Wannamaker LW, Matsen $\overline{\vec{n}}$ $\mathrm{JM}$, eds. Streptococci and streptococcal diseases: recognitions, understanding and management. New York: Academic Press, 1972:281-99.

${ }^{8}$ Culture negative endocarditis. Lancet 1978;ii:1164-5.

- Lehninger A. Biochemistry 2nd ed. New York: Wiley, 1976.

${ }^{10}$ Cayeux P, Acar JF, Chabbert YA. Bacterial persistence in streptococcal endocarditis due to thiol-requiring mutants. J Infect Dis 1971 ;124:247-54.

11 McCarthy LR, Bottone EJ. Bacteremia and endocarditis caused by satelliting streptococci. Am J Clin Pathol 1974; $61: 585-91$.

12 Diem K, ed. Documenta Geigy 7th ed. Manchester: CibaGeigy, 1979.

${ }^{13}$ Carey RB, Gross KC, Robert RB. Vitamin $B_{6}$ dependent Streptococcus mitior (mitis) isolated from patients with systemic infections. J Infect Dis 1974;131:722-6.

Requests for reprints to: Mr GS Tillotson, Department of Microbiology, Hope Hospital, Salford M6 8HD, England. 